\title{
Towards a Methodological Guide to share knowledge when creating Open Educational Resources
}

\author{
Julia Espinoza-Guzmán \\ TEC Digital \\ Tecnológico de Costa Rica \\ Cartago, Costa Rica \\ juliaespinoza@itcr.ac.cr
}

\author{
Liliana González \\ Universidad Tecnológica de León \\ Guanajuato, México \\ larredondo@utleon.edu.mx
}

\author{
Sergio Forero \\ Centro de Servicios \\ Crediticios, Colombia \\ sdforero@gmail.com
}

\author{
Ana María González \\ Institución Educativa \\ Buga, Colombia \\ anamgonzalezd@gmail.com
}

\begin{abstract}
Resumen-People claim the right to study and learn what they desire, when and where they want; for this reason it is necessary to offer flexible training methods that provide real opportunities for independent learning. In order to help solve this need, this article presents a methodological guide (MG) so people without experience or knowledge in software development can create, autonomously, Open Educational Resources (OER) executable in mobile equipment. In addition to MG, is presented a systematization process of experience and the implementation through a case study carried out in Mexico and Costa Rica. As a result participants created REA and appreciated the autonomy provided by the MG on average with 8 of 10 points.
\end{abstract}

Keywords-Guía Metodológica, App Inventor, Recursos Educativos Abiertos, autonomía.

\section{Introducción}

La tecnología, con su presuroso y constante cambio y con la posibilidad de una comunicación prácticamente instantánea, ha influido la conducta de los seres humanos [1], lo que sin duda también ha permeado el proceso de enseñanza y aprendizaje. En el ámbito educativo se plantea el derecho a la libertad, es decir, de manera creciente y generalizada "las personas pretenden poder trabajar, aprender y estudiar cuando quieran y desde donde quieran" [2]. Por otra parte, [3] el informe Hacia un Aprendizaje Universal plantea cifras alarmantes respecto a la educación global: 120 millones de niños no llegan a la escuela o la abandonan antes de su cuarto grado, 200 millones de jóvenes aunque terminan la secundaria no tienen las habilidades necesarias para desenvolverse en la vida o en el trabajo. La misma organización indica que el aprendizaje a lo largo de toda la vida es un imperativo global que necesita "ofrecer posibilidades flexibles de aprendizaje permanente en todos los ámbitos de la vida por medios formales, no formales e informales" [4]. Por otra parte, la sociedad actual demanda profesionales competentes para crear, difundir y evaluar situaciones de aprendizaje acordes con las exigencias del siglo XXI y que contribuyan a atender la necesidad de aprender que tienen las personas de la sociedad actual.
Además, muchos expertos tienen interés de contribuir con información y formación acerca de su área de conocimiento, lo que los convierte no solo en consumidores de información sino también en productores de conocimiento, este doble rol se denomina "prosumidores" [5] y [6]. Con la propuesta, que se presenta en este artículo, precisamente se pretende que la difusión del conocimiento de calidad no se circunscriba estrictamente a la academia, sino que se puede ampliar para que otros expertos compartan experiencias, conocimientos e información con aquellos que están ávidos de aprender.

Por otra parte, es en este ámbito donde las TIC puede contribuir, ya que juegan un rol fundamental por la cobertura que pueden lograr y por lo tanto pueden ser utilizadas "para apalancar el desarrollo socioeconómico de los países, así como su impacto en el bienestar de las sociedades." [7], o como lo dice [4] aprovechar el "potencial de las TIC para crear una nueva cultura del aprendizaje". Del mismo modo, el aprendizaje abierto y a distancia utilizando Recursos Educativos Abiertos (REA) representa "una nueva línea de aprendizaje que puede potenciar los resultados favorablemente en sus usuarios y son de gran ayuda para personas que viven en regiones económicamente desfavorecidas" [8]. Lo anterior se potencia, si además los REA se ofrecen a través de móviles debido a que permiten realizar la actividad formativa independientemente del espacio y tiempo, de forma que se ofrezca a cada persona una elección real en cuándo, cómo y dónde estudiar [9].

Si se unen los elementos mencionados: a) las necesidades de aprender, b) el interés por compartir conocimiento, c) los REA y d) la disponibilidad actual de equipos móviles, es posible hacer propuestas que promuevan el aprendizaje con flexibilidad espacial y temporal. Como una posible forma de atender esos requerimientos, los investigadores plantearon una Guía Metodológica para que, profesionales sin experiencia en desarrollo de software, tengan autonomía para crear REA de calidad ejecutables en móviles, de tal forma que aprovechen del "enorme potencial de contribuir para la mejoría de la calidad y de la eficacia de la educación.” [10]. En este artículo se presenta la GM, el proceso de sistematización de su uso y de la puesta en práctica por medio de un estudio de casos entre 2014 y 2016 en México y Costa Rica, con el fin de plantear un proceso incremental que permita 
aumentar el grado de autonomía para que profesionales de cualquier disciplina puedan crear REA para ser distribuidos en equipos móviles.

\section{Trabajo relacionado}

La UNESCO [11] indica que los recursos educativos abiertos (REA) son:

"Materiales de enseñanza, formativos o de investigación en cualquier soporte, digital o de cualquier otro tipo, que sean de dominio público o que hayan sido publicados bajo una licencia abierta que permita el acceso gratuito, así como el uso, modificación y redistribución por otros sin ninguna restricción o con restricciones limitadas."

En relación con los REA existen múltiples enfoques para abordarlos, por ejemplo propuestas para su búsqueda y selección según las situaciones de aprendizaje [12] y [13], se presentan repositorios para su almacenamiento y la localización [10], [12], [14], [15] y [16], las buenas prácticas en relación con su uso [17]. Otras corrientes estudian cómo los REA impactan la educación y el aprendizaje [10], [18], los aspectos pedagógicos [19], los procesos docentes para la producción de REA en espacios educativos formales [13] y [20], producción de REA y política pública [21]. También se localizan referencias acerca de ambientes de investigación educativa sobre los REA [18] y temas más avanzados como los recomendadores de Objetos de Aprendizaje [22]. También hay estudios integrales respecto a los $\mathrm{OA}$, que exponen enfoques pedagógicos para la creación, tecnologías para el desarrollo, procesos colaborativos para la creación y otros más [23].

Por su parte [24] expone que la OECD analiza un conjunto de temas relacionados con costo/beneficio, sostenibilidad en la producción de REA, derechos de propiedad intelectual, incentivos y barreras, fomentando que las universidades entreguen sus materiales para iniciativas relacionadas con REA, todo en el ámbito de educación superior. [25] Documenta un conjunto de experiencias realizadas en Europa y en Latinoamérica, respecto a los REA, y expone sobre aseguramiento de la calidad, sobre la formación de docentes, estándares en REA, políticas de alto nivel, descriptores (metadatos) que serán utilizados por los buscadores y metabuscadores que buscan localizar los REA y como "tomar decisiones informadas sobre la aplicabilidad, los desafíos y los beneficios de los REA".

Ahora bien, en el tema de tecnologías digitales y específicamente ante la gran oferta de celulares, los investigadores tomaron la decisión de centrarse en los que cuentan con el Sistema Operativo (SO) Android, ya que tienen una mayor participación en el mercado con más del 80 por ciento de éste ${ }^{1}$. Para programar los REA para dispositivos móviles con Android, se seleccionó App Inventor ${ }^{2}$ por ser software libre desarrollado en Google Education y se ejecuta como un servicio Web que es administrado por el Massachusetts Institute of Technology (MIT) y "permite a principiantes

1. http://www.gartner.com/newsroom/id/3215217

2. http://appinventor.mit.edu/explore/index-2.html y no desarrolladores de software creen aplicaciones para celulares y para tabletas" [26].

La interfaz iconográfica de App Inventor (ver figura 1) utiliza un editor de bloques que encajan unos con otros, lo que facilita a personas no programadoras, la comprensión de sentencias de software y porque permite concentrarse en la lógica de programación en vez de la sintaxis, disminuyendo así la carga cognitiva del desarrollador.

\section{Guía Metodológica y proceso de sistemati- zación para crear REA para móviles}

Para atender la necesidad de aprender y de compartir conocimiento, los investigadores proponen una GM para que profesionales sin experiencia en desarrollo de aplicaciones computaciones puedan crear REA ejecutables en equipos móviles con sistema operativo Android. No obstante, el objetivo va más allá de entregar esta GM a la comunidad profesional. Se propone además un proceso de sistematización de la experiencia del uso de la GM. De acuerdo con [27] sistematizar una experiencia es "entender por qué ese proceso se está desarrollando de esa manera, entender e interpretar lo que está aconteciendo, a partir de un ordenamiento y reconstrucción de lo que ha sucedido en dicho proceso." Lo relevante en este sentido es que la sistematización no se queda únicamente en describir o recopilar información de lo acontecido sino que "El eje principal de preocupación se traslada de la reconstrucción de lo sucedido y el ordenamiento de la información, a una interpretación crítica de lo acontecido para poder extraer aprendizajes que tengan una utilidad para el futuro." [27]. Con el proceso de sistematización propuesto se pretende aprender críticamente de la experiencia de uso de la GM para lograr: a) introducir mejoras en la práctica y b) dar a conocer los aprendizajes que pueden tener experiencias similares [27].

Entonces la GM y el proceso de sistematización (ver figura 2) se unen para colaborar con el "mejoramiento de experiencias en marcha o para facilitar la realización de nuevos ejercicios partiendo del desarrollo metodológico alcanzado durante la experiencia precedente." [28]. Los pasos para la sistematización son:

1. Identificación de la necesidad que se desea atender. En este caso compartir: a) conocimiento de calidad y b) el derecho de aprender y estudiar desde cualquier lugar y momento.

2. Redacción de la Guía Metodológica propiamente dicha para orientar la ejecución del proceso: en este caso la construcción de los REA para móviles, para efectos prácticos se denomina GM.

3. Realización de un estudio de casos en México y Costa Rica.

4. Llevar a cabo la reconstrucción metodológica y una validación técnica. Luego se hace el análisis cualitativo y cuantitativo de la información 


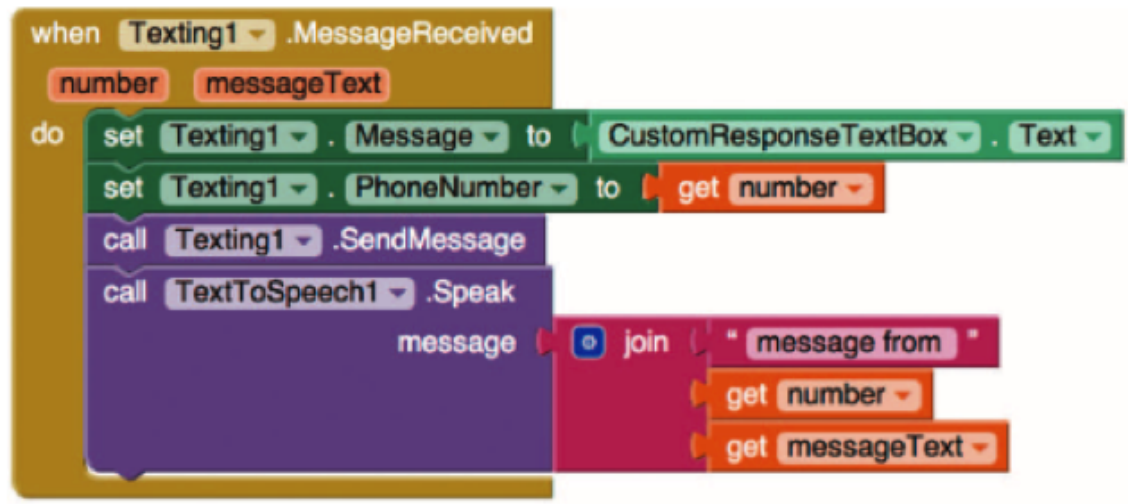

Figura 1. Ejemplo de bloques de programación en App Inventor. Fuente: Wolber, Abelson, Friedman (2015)

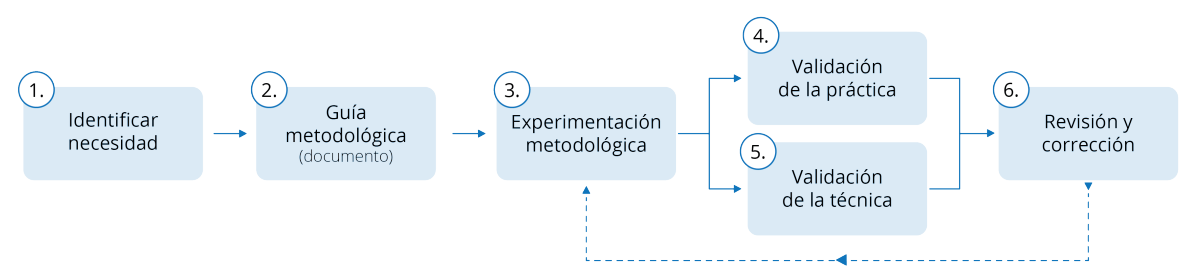

Figura 2. Proceso de sistematización del uso de la GM para crear REA. Fuente: Construcción propia, basada en [28]

5. Generar la versión 2 de la GM que contenga las mejoras nacidas de la experimentación, para promover una investigación a mayor escala.

\subsection{Guía Metodológica para crear REA}

La propuesta está dirigida a profesionales de cualquier disciplina del saber, y no se circunscribe a docentes aunque puede ser usada para fines académicos. Los REA, que se puedan desarrollar, siguiendo la GM, pueden estar enfocados en públicos diversos: agricultores, amas de casa, educadores, médicos, ingenieros, economistas, niños, jóvenes y adultos, en fin, cualquier persona que esté interesada en aprender. Los productos creados con ayuda de la GM pueden ser utilizados en la educación formal o informal, ya que se pueden combinar símbolos, vídeos, sonidos y otros recursos multimedia, lo cual cumple con las recomendaciones de la Agenda Regional de Prácticas Educativas Abiertas (OEP, s.f., p.3), que sugiere, entre otros aspectos, que "incorporen un enfoque abierto a la producción de conocimiento en la educación, promoviendo el uso, reutilización y remezcla de recursos educativos".

Uno de los pilares fundamentales con que se pensó la GM para crear REA, es lograr que el creador tenga el mayor nivel de autonomía posible que que la Real Academia de la Lengua Española define como "Condición de quien, para ciertas cosas, no depende de nadie" (RAE, 2001).
Por esto, uno de los objetivos dell estudio es determinar la influencia que tuvo el uso de la GM en profesionales de diversas áreas, y concretamente en la posibilidad de desarrollar autonomía en la creación de aplicaciones REA para dispositivos móviles Android.

Para brindar la autonomía, la GM describe las fases, tareas y entregables para desarrollar satisfactoriamente un REA en App Inventor. La GM cuenta con un conjunto de cinco fases (ver figura 3), un instructivo de uso, una guía de instalación de App Inventor y un guión para planificar y diseñar el material educativo. Además, el material de apoyo se complementó con un ejemplo ya desarrollado de un REA en un archivo .apk ${ }^{3}$ para instalar en un móvil Android. Todo se integró en un instructivo con las indicaciones, recursos, productos esperados del proceso e información de contacto del equipo de investigación.

En el cuadro 1 se presentan las fases de la GM, el objetivo y el producto entregable de cada una.

\section{Metodología de investigación}

En esta sección se describe la metodología que se utilizó para realizar el estudio acerca de la autonomía de la GM para crear REA. La propuesta de investigación parte del siguiente cuestionamiento general: ¿la guía metodológica desarrolló

3. Android Application Package 


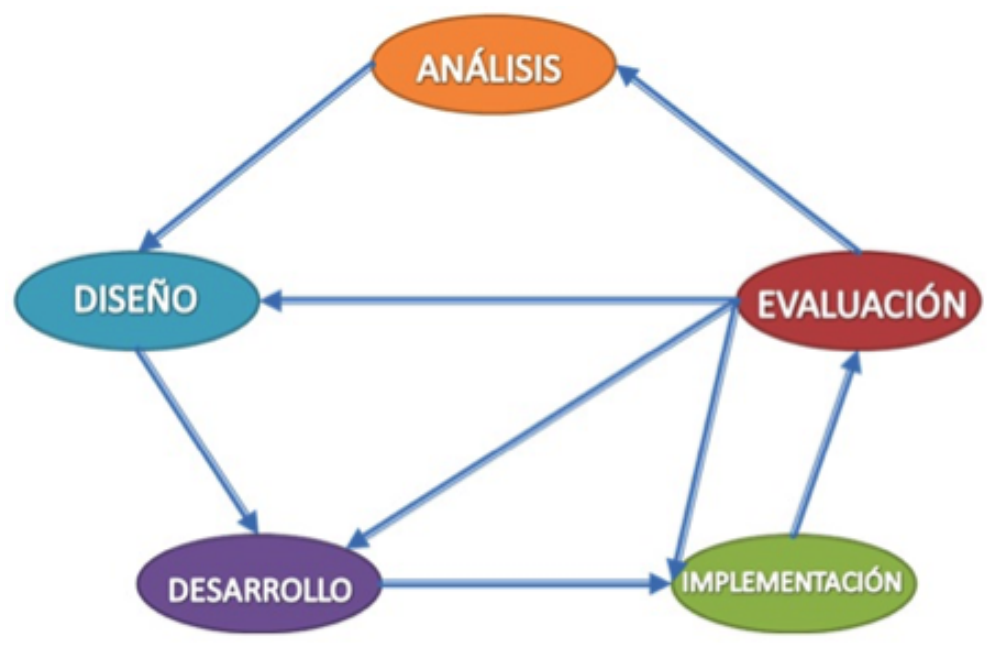

Figura 3. Fases de la Guía Metodológica para crear REA.Fuente: GM para crear REA. Fuente: construcción propia

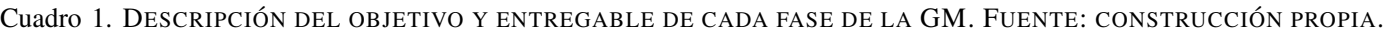

\begin{tabular}{|c|c|c|}
\hline Fase & Objetivo & Entregable \\
\hline Análisis & $\begin{array}{l}\text { Define área o problema que se quiere atender, } \\
\text { identifica requerimientos y recursos con que } \\
\text { cuenta para crear el REA }\end{array}$ & $\begin{array}{l}\text { Estructura jerárquica y conceptual, árbol de activi- } \\
\text { dades, Modelo de secuencia, navegación, Árbol de } \\
\text { actividades y recursos a utilizar. }\end{array}$ \\
\hline Diseño & Diseño de la interfaz gráfica y bosquejo del REA & Esbozo gráfico del REA y el guión técnico del REA. \\
\hline Desarrollo & $\begin{array}{l}\text { Utiliza App Inventor, incluye los recursos, hace } \\
\text { la estructura de navegación y sus componentes, } \\
\text { todo de acuerdo con el guión creado anterior- } \\
\text { mente. }\end{array}$ & $\begin{array}{l}\text { Estructura básica del REA, con recursos y nave- } \\
\text { gación funcional. }\end{array}$ \\
\hline Implementación & $\begin{array}{l}\text { Integra todos los recursos en App Inventor, em- } \\
\text { paquetar y probar el REA en un emulador o en } \\
\text { el celular. }\end{array}$ & El REA instalado y probado en el equipo móvil. \\
\hline Evaluación & Valida que cumple el objetivo propuesto & $\begin{array}{l}\text { El REA validado con público real, considerando } \\
\text { cumplimiento del objetivo educativo, eficacia, fa- } \\
\text { cilidad de navegar por el recurso de forma factible } \\
\text { y cómoda, usabilidad. }\end{array}$ \\
\hline
\end{tabular}

autonomía en los profesionales participantes para la creación de aplicaciones REA con App Inventor en tecnología Android, con la finalidad de mejorar los procesos de enseñanza y aprendizaje? Para efectos del proyecto, los participantes deben contar con competencias digitales que les permitan aplicar las TIC como parte de su ejercicio profesional.

La investigación se abordó por medio de un estudio de casos - descriptivo, por las siguientes razones: a) verificar y tener un enfoque acerca de la utilidad y apropiación por parte de los participantes respecto a la utilización de la GM, b) comprender un REA en una situación particular, c) estudiar de manera empírica acerca de una situación específica en un contexto de la vida real, d) recolectar datos acerca de diferentes variables de interés y, e) permitir investigar, en este caso, a un grupo de individuos que es visto como una entidad sobre la cual se espera conocer la percepción e influencia acerca de la GM para elaborar el REA y sus constructos, de tal forma que pueda aportar experiencia y conocimientos relacionados con los REA en equipos móviles [29] y [30]. El estudio se realizó con tres profesionales de áreas diferentes entre sí y un estudiante de educación superior. La conformación del grupo es la siguiente: una persona de diseño publicitario, una doctora en nutrición, un profesional en tecnologías de la información y un estudiante de administración de tecnología de información. Sus edades están entre los 21 y 32 años, de las ciudades de León, México; Cartago y Corcovado, Costa Rica. De esta manera, se utilizó un estudio de casos simple, ya que el interés inicial radicó en obtener información acerca de la versión inicial de la GM con el fin de validar su facilidad de uso, comprensión y la factibilidad de utilizarla en la construcción de nuevos REA por parte de los participantes.

Para este efecto, contaron con un estimado de 12 horas en un plazo de ocho días naturales en que debían cumplir con todas las fases, completar instrumentos y desarrollar la aplicación en App Inventor. Estas restricciones de tiempo se 
establecieron para determinar el nivel de autonomía que se lograría bajo estas condiciones.

En este sentido se identificaron dos constructos: GM y tecnología móvil; además para la obtención de la información se construyeron dos instrumentos: una encuesta y la entrevista semi-estructurada, que previamente, fueron validadas por un grupo de cuatro expertos de México y Colombia. El principal objetivo de la encuesta fue conocer las características personales y de conocimiento de software para aplicaciones móviles en los participantes en la investigación; así mismo la entrevista semi-estructurada se realizó al final de la investigación para conocer la utilidad que tuvo la guía metodológica, ventajas y áreas de mejora al crear una aplicación móvil. Con la finalidad de disminuir amenazas en la recolección y validez de los datos, en ambos instrumentos se informó a los participantes el propósito de la investigación, para concientizarlos de su importancia y garantizando que se respetaría la confidencialidad de los datos con la intención de obtener un producto de calidad.

Para la obtención de resultados se utilizó la información de dichos instrumentos y la observación de los dos productos generados por los participantes: el guión completo para un REA del área de especialidad y el REA creado en el programa App Inventor (archivo apk) que se instaló en un celular Android. Todos estos elementos se utilizaron como insumos para realizar la triangulación de datos, de métodos y de investigación teórica [31].

\section{Resultados}

Cada uno de los cuatro participantes logró desarrollar un REA que contiene experiencias educativas relacionadas con procesos cotidianos. Así se obtienen cuatro productos que se presentan en el Cuadro 2.

Cuadro 2. DESCRIPCIÓN DEL OBJETIVO Y ENTREGABLE DE CADA FASE DE LA GM

\begin{tabular}{|l|l|}
\hline Área de especialidad & Producto \\
\hline \hline Diseño gráfico & $\begin{array}{l}\text { Reglas básicas de comunicación y diseño } \\
\text { para crear un arte. }\end{array}$ \\
\hline Nutrición & $\begin{array}{l}\text { Guía para que los pacientes identifiquen las } \\
\text { porciones nutricionales }\end{array}$ \\
\hline $\begin{array}{l}\text { Tecnologías de Informa- } \\
\text { ción }\end{array}$ & $\begin{array}{l}\text { Antenas HD, realización de una antena con } \\
\text { componentes caseros para la captación de } \\
\text { señales digitales libres. }\end{array}$ \\
\hline Estudiante universitario & $\begin{array}{l}\text { Guía turística de preparación para la visi- } \\
\text { ta al Parque Nacional Corcovado en Costa } \\
\text { Rica, zona de origen del estudiante. }\end{array}$ \\
\hline \multirow{2}{*}{ Fuente: construcción propia. }
\end{tabular}

A partir de la información recolectada en la encuesta, la entrevista y del análisis documental de los productos, se procedió a hacer la sistematización y el análisis crítico de los resultados. A partir de esos procesos, se determinó que, prácticamente todos los REA creados incluyen imágenes, etiquetas, vídeos, botones para la navegación y es posible descargarlos para distribuirlos en móviles. Las figuras 4 y 5 muestran parte de los productos generados por dos de los participantes.

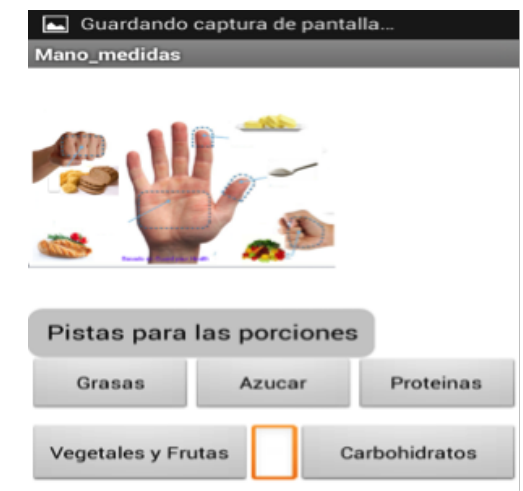

Figura 4. Captura de pantalla del REA (archivo apk) del participante 2

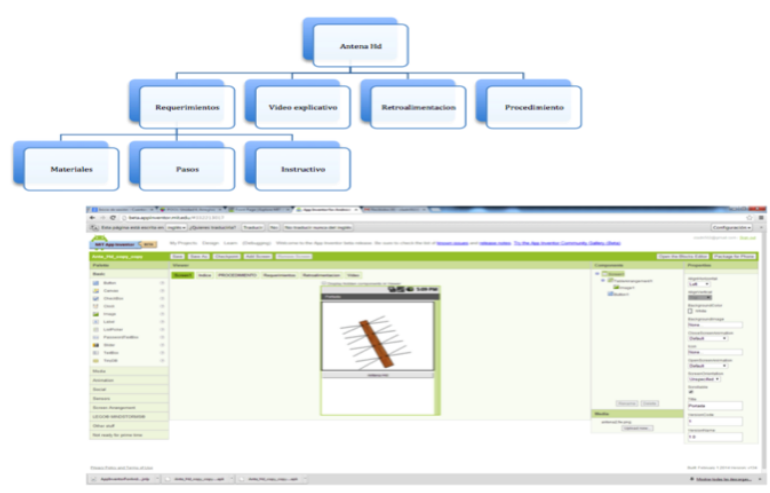

Figura 5. Captura de pantalla de construcción en App Inventor, participante 3

Finalmente, los participantes manifestaron que el tiempo para el desarrollo de la aplicación fue el principal inconveniente, inclusive por encima de la dificultad técnica. Por lo tanto, se recomienda realizar este ejercicio en un periodo más extenso

\subsection{Resultados obtenidos de la guía metodológica}

Las personas participantes afirmaron que la GM les suministró los pasos fundamentales para analizar y diseñar un REA básico para dispositivos móviles Android, los condujo a hacer un análisis detallado y profundo de necesidades informativas y les permitió generar un diseño ajustado y preciso de los requisitos previamente establecidos. Así mismo, indicaron que este material es comprensible y que les permitió el desarrollo, la implementación y evaluación del REA creado. Cada participante logró desarrollar el hito guión, al especificar el objetivo de aprendizaje, los contenidos, la ruta de navegación, el diseño de pantallas y la ubicación de los de recursos de apoyo.

Sin embargo, en la fase de desarrollo, la mayoría de personas bajo investigación, manifestó necesitar ayuda técnica con la puesta en marcha de App Inventor. Esto debido a que, la instalación del software requiere de conocimientos básicos en el área de informática o porque era la primera 
vez que usan App Inventor. Por consiguiente, en la cuarta fase de la GM, implementación, prácticamente todos los casos necesitaron de ayuda técnica para generar el producto esperado: un archivo con extensión apk. Igualmente, al evaluar los REA desarrollados, se encontró que tres personas lograron llevar a cabo todos los pasos propuestos en la GM. Una de ellas tuvo inconvenientes con aspectos técnicos que, con un poco más de tiempo, se pudieron haber solventado. En la fase de evaluación del REA creado, en promedio los sujetos indagados calificaron con ocho puntos el producto que construyeron.

La experiencia llevada a cabo les permitió calificar la autonomía al aplicar la GM, con un nivel medio-alto (8 de 10 puntos posibles). Una causa probable para no obtener la puntuación máxima fue el poco tiempo de apropiación de la GM y la necesidad de más apoyo técnico durante la instalación de App Inventor e implementación del REA. A pesar de esto, ellos indicaron que resultó enriquecedor poder desarrollar un REA de su área de especialidad sin ninguna experiencia previa en este software.

\subsection{Resultados obtenidos en el constructo de tec- nología móvil}

En el constructo de tecnología móvil se pudo observar que ninguno de los participantes tenía experiencia previa en realizar aplicaciones de este tipo, así como tampoco tenían conocimiento de un software que se utilizado para esto. Sin embargo, todos demostraron confianza en sus habilidades con el uso de las TIC para desarrollar una aplicación que les sirvió como apoyo en sus propósitos, basados en el uso de una tecnología emergente.

Por otra parte, los participantes afirmaron que el software App Inventor es una excelente herramienta iconográfica que les permitió el desarrollo de la aplicación móvil, puesto que, no se necesita conocer sobre programación para crear pantallas, incluir recursos y aplicar estructuras lógicas y de navegación. Finalmente, manifestaron que volverían a utilizarlo, convencidos de que con práctica se pueden lograr aplicaciones de mayor calidad. Adicionalmente, uno de los participantes mencionó que una de las desventajas presentadas es que el software no es intuitivo, por lo que fue necesario recurrir al ejemplo completo, proporcionado por el equipo de investigación, para apoyarse en el proceso ahí descrito. Asimismo, en los casos que lo requirieron los desarrolladores acudieron a los investigadores para resolver las dudas que se les presentaron; además, todos coincidieron que faltó tiempo para apropiarse de los conceptos de la GM y poder familiarizarse con la aplicación.

\subsection{Análisis de los resultados}

El primer aspecto que se encontró en el presente estudio, se refiere a la autonomía desarrollada por los participantes en la realización de un REA, puesto que, al aplicar las recomendaciones de las fases de análisis, diseño y desarrollo de la GM, lograron generar una aplicación que se pudo instalar en un celular Android. Cabe resaltar este aspecto puesto que todos lograron el producto esperado en App Inventor, aún los que no tenían experiencia previa en programación.

Todas las personas objeto de este estudio coincidieron en que la principal dificultad fue la falta de tiempo para asimilar y comprender mejor la GM, lo que impidió desarrollar hitos de mayor calidad tales como: el guión y el archivo ejecutable del REA (.apk). Esto se refleja en los inconvenientes presentados por los participantes que no lograron apropiarse totalmente del software de desarrollo (App Inventor); por esta razón, se necesitó ayuda adicional por parte del equipo de investigadores, tanto para la descarga e instalación del software, como en la atención de consultas técnicas, pero como lo indicó [32] el aprendizaje significativo requiere un periodo para tener el efecto esperado.

Por otra parte, el análisis documental de los productos generados por los participantes demostró que el guión se ajustó a los lineamientos elementales establecidos en la GM, de ahí se desprende que se haya realizado un análisis análisis elemental de necesidades, un diseño básico de navegación a través de las pantallas y un modesto desarrollo del REA. Los hallazgos expuestos en cuanto al tiempo y la apropiación tecnológica son importantes, ya que demostraron que los participantes bajo investigación requieren de un tiempo prudencial para dominar la herramienta tecnológica y lograr una impecable implementación y evaluación del REA.

\section{Conclusiones}

La sistematización de la experiencia al utilizar la GM permitió recopilar información y detectar mejoras que pueden ser aplicadas en un futuro cercano. Ya que, como indica [33] citado por [34] acerca de las guías metodológicas "cumplen una función particularmente útil para contribuir al mejoramiento de experiencias en marcha o para facilitar la realización de nuevos ejercicios." En este caso, la GM ayuda a los participantes a crear material que puede ser compartido y distribuido para enriquecer el aprendizaje en cualquier lugar y momento, de acuerdo a las expectativas de las personas ante los retos del siglo XXI. La metodología propuesta permite seguir probando la GM y ofrecer mejoras sustanciales para que profesionales de cualquier área puedan generar REA de calidad y cumplir con los pasos expuestos por [27] respecto a la sistematización de experiencias como un proceso para mejorar la práctica.

En función de los objetivos de la investigación y de los resultados, se puede decir que la GM influyó positivamente en el desarrollo de la autonomía para crear aplicaciones y REA para móviles Android, debido a que los participantes señalaron, en promedio, que la GM les les brindó un nivel de autonomía de 8 puntos de 10 posibles. Esto coincide con experiencias previas, las cuales que demuestran que para los usuarios no expertos, es posible elaborar una lógica sencilla para que puedan construir aplicaciones en App Inventor [35] y [36].

A partir de los hallazgos, el siguiente paso es generar una versión depurada de la GM que considere las observaciones dadas por los participantes y también con las que detectaron 
los mismos investigadores. A partir de esto es posible hacer una investigación con mayor cantidad de profesionales y en diversos ambientes, lo que a su vez permitiría medir con mayor precisión el nivel de autonomía logrado.

Se recomienda aplicar el proceso de puesta en práctica y sistematización para compartir conocimiento de calidad porque: a) "Hay todavía un escenario muy amplio para explorar en donde los objetos de aprendizaje encontrarán seguramente un espacio para aportar al fortalecimiento de la calidad" [19], b) se puede contribuir a lograr ese sueño de tener individuos formados y educados en la sociedad del conocimiento [37], c) no centra el conocimiento solamente en la academia, d) porque aprovecha "las CINCO R (5R) que permiten los dispositivos móviles: Registrar, Recuperar, Recordar, investigaR y Relacionar (se) con otros" [6] y e) los REA permiten internacionalizar el conocimiento en especial en América Latina por compartir un mismo idioma. Las ventajas de usar el proceso de sistematización y la GM van más allá debido a que puede constituirse en una forma de crear REA que permita a personas de diversos entornos culturales, económicos, sociales, educativos, técnicos y profesionales desarrollar autonomía y nuevas habilidades de competitividad, cooperación y colaboración en un espacio altamente creativo con la oportunidad de distribución en forma gratuita [24]. Además, los REA pueden ser eficientes para promover el aprendizaje a lo largo de la vida [24] y pueden mitigar la necesidad de aprendizaje, que como lo indica UNESCO es uno de los retos que pueden ser solventados con ayuda de las TIC. La utilización de la GM es una forma de promover la generación de conocimiento a través de REA, sobre todo entre profesionales que están dispuestos a crear y compartir con la sociedad y de esta forma suplir parte del requerimiento de las personas de "aprender y estudiar cuando quieran y desde donde quieran" [2].

La nueva versión de la GM tendrá aplicados los siguientes cambios:

1. Incluir elementos icónicos, así como hacer un sitio web que incluya recursos y enlaces a ejemplos resueltos, ayuda técnica para la instalación y un capítulo de preguntas frecuentes, con la finalidad de que el desarrollador logre mayor apropiación de la herramienta y así explotar al máximo los recursos con los que cuenta App Inventor.

2. Crear un infográfico con los pasos y tiempos estimados de dedicación para realizar las diferentes actividades, así como publicar en la web un e-book con la GM mejorada.

3. Agregar la licencia Creative commons para compartirla.

4. Incluir más ejemplos de REA creados con la GM.

5. Colocar estos recursos en un repositorio de acceso libre.

\section{Limitaciones}

En el ámbito Latinoamericano, el problema más importante sigue siendo "la conexión a internet y la infraestructura tecnológica," dicho en UNESCO en su sitio "La situación actual de los Recursos Educativos Abiertos a nivel mundial"

Se probó solamente con cuatro participantes, no obstante, la experimentación de campo permitió comprobar aspectos fundamentales e identificar mejoras factibles de aplicar en la GM.

\section{Trabajo futuro}

Una vez aplicadas las mejoras en el versión 2 de la GM, la tarea a corto plazo es probar el paquete metodológico en mayor cantidad de casos, temas y diversidad de habilidades de los creadores.

Otra labor es establecer un repositorio para almacenamiento y divulgación de los REA creados, de tal forma que se publiquen y faciliten el acceso para que el público meta pueda localizarlos y utilizarlos [10]. Esto lleva al tema de los metadatos que será un requisito en cada producto generado y que la GM debe incluir en las explicaciones.

Entre otras líneas de investigación relacionadas está la validación de la calidad de los REA y su contenido, analizar el uso que se da a los REA, la opinión que tienen los usuarios, si causan impacto positivo, abordar el tema de derechos de autor y el temor de los creadores a perder la propiedad intelectual [10].

Ampliar la GM para el desarrollo en cualquier dispositivo móvil y sistema operativo, con el firme objetivo de no encasillar al usuario por un dispositivo y software concreto, sino, de proveer a los participantes una libertad absoluta para desarrollar y visualizar su aplicación en cualquier medio móvil (usabilidad y accesibilidad). Para esto habrá que valorar el uso de herramientas híbridas que permitan desarrollar un mismo REA para móviles con sistemas operativos diferentes, pero posiblemente solo puede ser usado por personas con experiencia en ambientes de desarrollo de software. El aspecto favorable es que la GM contiene elementos de planificación pedagógica que pueden ser aplicados en otras tecnologías de desarrollo para llegar a proveer REA que puedan ejecutarse en cualquier dispositivo móvil.

Finalmente, la GM deberá incorporar un apartado en el cual se proporcione la información necesaria sobre el cómo evaluar la calidad y funcionalidad los REA generados y de qué forma podrían integrarse en los procesos de enseñanzaaprendizaje.

\section{Reconocimientos}

Los autores desean agradecer a los cuatro participantes en el estudio de casos, por su tiempo y realimentación que ofrecieron para mejorar la Guía Metodológica y el proceso de sistematización propuestos.

\section{Referencias}

[1] M. Soledad Ramírez Montoya, "Dispositivos de mobile learning para ambientes virtuales: implicaciones en el diseño y la enseñanza." Apertura: Revista de Innovación Educativa, vol. 8, no. 9, 2008. 
[2] E. Durall, B. Gros, M. Maina, L. Johnson, and S. Adams, "Perspectivas tecnológicas: educación superior en iberoamérica 2012-2017."

[3] UNESCO, "Hacia un Aprendizaje Universal," 2013. [Online]. Available: http://www.uis.unesco.org/Education/Documents/ lmtf-summary-rpt-sp.pdf

[4] O. UNESCO, "Position paper on education post-2015," 2014.

[5] M. C. Cantoni Briceño, "Mejorando la calidad de la educación a distancia a través del uso de recursos educativos abiertos," Los recursos de aprendizaje en la educación a distancia, p. 60.

[6] V. Chirino and A. Olvera, "El profesor como prosumidor en la administración del conocimiento para la mejora continua de recursos de aprendizaje," in Los Recursos de Aprendizaje en Educación a Distancia: Nuevos Escenarios, Experiencias y Tendencia, F. Ramírez and C. Rama, Eds. Lima, Perú: Universidad Alas Peruanas, 2014, pp. $45-58$.

[7] J. V. Burgos, "Distribución de conocimiento y acceso libre a la información con recursos educativos abiertos (rea)."

[8] D. Olcott Jr, "New pathways to learning: Leveraging the use of oers to support non-formal education," Revista de Universidad y Sociedad del Conocimiento, vol. 10, no. 1, pp. 327-344, 2013.

[9] J. Cabero, C. Llorente, and P. Román, "La tecnología cambió los escenarios: el efecto pigmalión se hizo realidad." Comunicar, vol. 15, no. 28, 2007.

[10] N. Butcher, A basic guide to open educational resources (OER). Commonwealth of Learning, Vancouver and UNESCO, 2015.

[11] UNESCO, "2012 WORLD OPEN EDUCATIONAL RESOURCES (OER) CONGRESS - Paris OER Declaration_01.pdf,” 2012. [Online]. Available: http://www.unesco.org/new/fileadmin/MULTIMEDIA/HQ/ CI/CI/pdf/Events/Paris\%20OER\%20Declaration_01.pdf

[12] F. Mortera, A. Salazar, J. Rodríguez, and J. A. Pérez, "Guía de referencia para el uso de recursos educativos abiertos (rea) y objetos de aprendizaje (oa)," Montemorelos, Nuevo León, México: CUDICONACYT, 2011.

[13] M. Acuña, M. E. Gil, and A. M. Sandoval, "Buenas prácticas para la selección de recursos educativos abiertos: experiencias del mooc innovación educativa con rea," Revista Actualidades Investigativas en Educación, vol. 16, no. 2, 2016.

[14] J. Díaz, A. Schiavoni, A. P. Amadeo, M. E. Charnelli, J. G. Schulz, and A. Humar, "Integrando un repositorio digital de objetos de aprendizaje con servicios que promuevan su uso y mantenimiento," Conferencias LACLO, vol. 5, no. 1, 2015.

[15] G. Santos-Hermosa, N. Ferran-Ferrer, and E. Abadal, "Recursos educativos abiertos: repositorios y uso," El profesional de la información, vol. 21, no. 2, pp. 136-145, 2012.

[16] F. J. Mortera, "Implementación de recursos educativos abiertos a través del portal TEMOA del tecnológico de monterrey, méxico," Formación universitaria, vol. 3, no. 5, pp. 9-20, 2010.

[17] F. Mortera, "Buenas prácticas para el uso académico de recursos educativos abiertos y objetos de aprendizaje," in Proceedings del XVI Congreso EDUTEC 2013. San José, Costa Rica: EDUTEC, 2013.

[18] M. S. Ramírez Montoya and J. V. Burgos Aguilar, "Recursos Educativos abiertos en ambientes enriquecidos con Tecnología: Innovación en la práctica educativa," 2010. [Online]. Available: http://catedra.ruv.itesm.mx/bitstream/987654321/566/8/ebook

[19] A. Chiappe Laverde, "Acerca de lo pedagógico en los objetos de aprendizaje-reflexiones conceptuales hacia la construcción de su estructura teórica," Estudios pedagógicos (Valdivia), vol. 35, no. 1, pp. 261-272, 2009.

[20] M. F. Maina and L. Guàrdia Ortiz, "Un modelo de creación de contenidos en abiertos para el aprendizaje," Maina, 2012.
[21] D. Vila-Viñas, D. Araya, and P. Bouchard, "Educación: recursos educativos abiertos," Buen Conocer-FLOK Society. Modelos sostenibles y políticas públicas para una economía social del conocimiento común y abierto en el Ecuador. Quito: IAEN-CIESPAL. Recuperado a partir de http://book. floksociety. org/ec/1/1-1-educacion-recursoseducativos-abiertos, 2015.

[22] J. Solís, M. Chacón-Rivas, and C. Garita, "Agente híbrido recomendador de objetos de aprendizaje," Conferencias LACLO, vol. 5, no. 1, 2015.

[23] A. PEA, "Un enfoque de abajo hacia arriba en américa latina y europa para desarrollar un espacio común de educación superior,' PEA, Report, s.f.

[24] J. Hylén and T. Schuller, "Giving knowledge for free," Organisation for Economic Cooperation and Development. The OECD Observer, no. 263 , p. $21,2007$.

[25] A. Inamorato, C. Cobo, and C. Costa, "Open educational resources: Cases from latin america and europe in higher education," Compendium-Open Educational Resources: Cases from Latin America and Europe in Higher Education, Andreia Inamorato dos Santos, Cristóbal Cobo, Celso Costa, Niterói: CEAD-UFF, 2012.

[26] D. Wolber, H. Abelson, and M. Friedman, "Democratizing computing with app inventor," GetMobile: Mobile Computing and Communications, vol. 18, no. 4, pp. 53-58, 2015. [Online]. Available: http://dl.acm.org/citation.cfm?id=2721935

[27] O. Jara, "La sistematización de experiencias: práctica y teoría para otros mundos posibles," San José, Costa Rica: CEP Alforja, 2012.

[28] E. Oñate, "Metodológica en gestión de riesgos y desastres para la unidad educativa luxemburgo de educación general," 2015.

[29] R. K. Yin, Case study research: Design and methods. Sage publications, 2013.

[30] R. E. Stake, Investigación con estudio de casos. Ediciones Morata, 2007.

[31] R. Hernández Sampieri, C. Fernández Collado, and P. Baptista Lucio, Metodología de la investigación. McGraw-Hill, 2010, vol. 1.

[32] M. L. Rodríguez Palmero, "Teoría del aprendizaje significativo," 2004.

[33] M. A. Martínez, "Las guías metodológicas: Orientaciones para su preparación,” ICADE, 2001.

[34] A. Aguilar, S. P. Güity, and J. R. Hurtado, “diseño y validación de guías metodológicas para el desarrollo de competencias del segundo nivel de educación básica,' Investigación educativa en la UPNFM: 2006-2010, pp. 166-171, 2011.

[35] A. Fero McKinney, "Yes, you can create your own mobile apps!" Clavier Companion, vol. 5, no. 5, 2013.

[36] Y.-C. Hsu, K. Rice, and L. Dawley, "Empowering educators with google's android app inventor: An online workshop in mobile app design," British Journal of Educational Technology, vol. 43, no. 1, pp. E1-E5, 2012.

[37] S. I. Adame Rodríguez, L. Lloréns Baez, and M. Schorr Wiener, "Retrospectiva de los repositorios de acceso abierto y tendencias en la socialización del conocimiento," Revista electrónica de investigación educativa, vol. 15, no. 2, pp. 148-162, 2013. [Online]. Available: http://www.scielo.org.mx/scielo.php?pid= S1607-40412013000200010\&script=sci_arttext 